\title{
Assessing the pharmacokinetic profile of the CamMedNP natural products database: an in silico approach
}

\author{
Fidele Ntie-Kang ${ }^{1,2,3^{*}}$, James A Mbah ${ }^{4}$, Lydia L Lifongo ${ }^{2}$, Luc C Owono Owono ${ }^{1,5}$, Eugene Megnassan ${ }^{6}$, \\ Luc Meva'a Mbaze', Philip N Judson ${ }^{8}$, Wolfgang Sippl ${ }^{3}$ and Simon MN Efange ${ }^{4}$
}

\begin{abstract}
Background: Drug metabolism and pharmacokinetic (DMPK) assessment has come to occupy a place of interest during the early stages of drug discovery today. Computer-based methods are slowly gaining ground in this area and are often used as initial tools to eliminate compounds likely to present uninteresting pharmacokinetic profiles and unacceptable levels of toxicity from the list of potential drug candidates, hence cutting down the cost of the discovery of a drug.

Results: In the present study, we present an in silico assessment of the DMPK profile of our recently published natural products database of 1,859 unique compounds derived from 224 species of medicinal plants from the Cameroonian forest. In this analysis, we have used 46 computed physico-chemical properties or molecular descriptors to predict the absorption, distribution, metabolism and elimination (ADME) of the compounds. This survey demonstrated that about $50 \%$ of the compounds within the Cameroonian medicinal plant and natural products (CamMedNP) database are compliant, having properties which fall within the range of ADME properties of $>95 \%$ of currently known drugs, while $>73 \%$ of the compounds have $\leq 2$ violations. Moreover, about $72 \%$ of the compounds within the corresponding 'drug-like' subset showed compliance.

Conclusions: In addition to the previously verified levels of 'drug-likeness' and the diversity and the wide range of measured biological activities, the compounds in the CamMedNP database show interesting DMPK profiles and, hence, could represent an important starting point for hit/lead discovery from medicinal plants in Africa.
\end{abstract}

Keywords: ADMET; Database collection; Descriptors; In silico; Medicinal plants; Natural products

\section{Background}

Natural products (NPs) play an increasingly important role in drug discovery today [1-5], both serving as drugs and as templates for the design of nature-inspired medicines $[3,6]$. In fact, it has been reported that a significant proportion of drugs that undergo clinical trials are either naturally occurring or are derived from NPs [7]. What characterises NPs are their richness in stereogenic centres and coverage of segments of chemical space which are typically not occupied by a majority of synthetic molecules

\footnotetext{
* Correspondence: ntiekfidele@gmail.com

'CEPAMOQ, Faculty of Science, University of Douala, P.O. Box 8580, Douala, Cameroon

${ }^{2}$ Chemical and Bioactivity Information Centre, Department of Chemistry, Faculty of Science, University of Buea, P.O. Box 63, Buea, Cameroon Full list of author information is available at the end of the article
}

and drugs $[8,9]$. In addition, they generally contain more oxygen atoms and less aromatic atoms on average, when compared with 'drug-like' molecules [8-11]. It is needless to say that NPs sometimes fail the famous 'drug-likeness' test due to the often bulky nature of naturally occurring metabolites [11].

It is also worth mentioning that designing drug-like molecules having interesting pharmacokinetic properties is an important paradigm in drug discovery programs $[12,13]$. This entails the search for lead compounds which can be easily orally absorbed, easily transported to their desired site of action, not easily attacked by metabolising enzymes so as to form toxic metabolic products before reaching the targeted site of action and easily eliminated from the body before accumulating in sufficient amounts

\section{实}


that may produce adverse side effects. The ensemble of the above properties is often referred to as absorption, distribution, metabolism and elimination (ADME) properties, or better still ADMET or ADME/T or ADMETox (i.e. if toxicity criteria are also taken into consideration).

Computer-based in silico approaches for the prediction of ADMET profiles of drug leads at early stages of drug discovery are increasingly gaining ground [14-16]. This could be explained by the relative cost advantage added to the time factor, when compared to standard experimental approaches for ADMET profiling $[17,18]$. On these grounds, several theoretical methods for the determination of ADMET parameters have been developed and implemented in a number of currently available software for drug discovery protocols [19-22], even though the predictions are sometimes disappointing [23]. Such software often make use of quantitative structure-activity relationships [22-24] or knowledge-base methods [25-27]. The goal has been to considerably cut down on the currently very high cost of discovery of a drug [17]. A promising lead is often defined as a compound which combines potency with an admirable ADMET profile. As such, compounds with unfavourably predicted pharmacokinetic profiles are either completely dismissed from the list of potential drug candidates (even if they prove to be highly potent) or the drug metabolism and pharmacokinetics (DMPK) properties are 'fine tuned' in order to improve their chances of making it to clinical trials [28]. This explains why the 'graveyard' of very highly potent compounds which do not make it to clinical trials keeps filling up, to the extent that the process of drug discovery often presents the challenge of either resorting to new leads or 'resurrecting' some buried leads with the view of fine-tuning their ADMET profiles.

In a recent paper, we have presented a database of 1,859 compounds derived from the Cameroonian flora, Cameroonian medicinal plant and natural products (CamMedNP), the compounds being predicted to be sufficiently orally available and diverse to be employed in lead discovery programs [29]. Additional arguments in favour of the use of this database are the wide range of the previously observed biological activities of the compounds and the wide range of ailments being treated by traditional medicine with the help of the herbs from which the compounds have been derived $[29,30]$.

Numerous drugs at a late stage of pharmaceutical development and many more lead compounds fail due to adverse pharmacokinetic properties [18]. It is, therefore, important to incorporate the prediction of the ADME properties into the lead compound selection, by means of molecular descriptors. A molecular descriptor is often defined as a structural or physico-chemical property of a molecule or part of a molecule, for example the logarithm of the $n$-octanol/water partition coefficient $(\log P)$, molar weight $(\mathrm{MW})$ and total polar surface area. A number of relevant molecular properties (descriptors) are often used to help predict the pharmacokinetic behaviour of potential drug leads. In the present study, we have carried out an in silico assessment of the ADMET profile of the CamMedNP database by the use of computed molecular descriptors currently implemented in a wide range of software tools as indicators of the pharmacokinetic properties of a large proportion of currently known drugs.

\section{Methods}

\section{Data sources and generation of 3D structures}

The plant sources, geographical collection sites, chemical structures of pure compounds and their measured biological activities were retrieved from literature sources and have been previously described [29]. The three-dimensional (3D) structures were generated using the builder module of MOE [31], and energy minimization was subsequently carried out using the MMFF94 [32] until a gradient of $0.01 \mathrm{kcal} / \mathrm{mol}$ was reached.

\section{Initial treatment of chemical structures and calculation of ADMET-related descriptors}

The 1,859 low-energy 3D chemical structures in the CamMedNP library were saved in mol 2 format and initially treated with LigPrep [33], distributed by Schrodinger, Inc. (New York, USA). This implementation was carried out with the graphical user interface of the Maestro software package (New York, USA) [34], using the OPLS force field [35-37]. Protonation states at biologically relevant $\mathrm{pH}$ were correctly assigned (group I metals in simple salts were disconnected, strong acids were deprotonated and strong bases protonated, while topological duplicates and explicit hydrogens were added). All molecular modelling was carried out on a Linux workstation (San Francisco, USA) with a $3.5 \mathrm{GHz}$ Intel Core2 Duo processor (Santa Clara, USA). A set of the ADMET-related properties (a total of 46 molecular descriptors) were calculated using the QikProp program (New York, USA) [21] running in normal mode. QikProp generates physically relevant descriptors and uses them to perform ADMET predictions. An overall ADMEcompliance score, drug-likeness parameter (indicated by \#stars), was used to assess the pharmacokinetic profiles of the compounds within the CamMedNP library. The \#stars parameter indicates the number of property descriptors computed by QikProp, which falls outside the optimum range of values for $95 \%$ of known drugs. The methods implemented were developed by Jorgensen et al. [38-40]. Among the calculated descriptors are the total solventaccessible molecular surface, $S_{\text {mol }}$ in $\AA^{2}$ (probe radius 1.4 $\AA$; range for $95 \%$ of drugs is 300 to $1,000 \AA^{2}$ ); the hydrophobic portion of the solvent-accessible molecular surface, $S_{\text {mol,hfob }}$ in $\AA^{2}$ (probe radius $1.4 \AA$; range for $95 \%$ of drugs is 0 to $750 \AA^{2}$ ); the total volume of molecule enclosed by solvent-accessible molecular surface, $V_{\text {mol }}$ in $\AA^{3}$ (probe 
radius $1.4 \AA$; range for $95 \%$ of drugs is 500 to $2,000 \AA^{3}$ ); the logarithm of aqueous solubility, $\log S_{\text {wat }}$ (range for $95 \%$ of drugs is -6.0 to 0.5 ) [36,38]; the logarithm of predicted binding constant to human serum albumin, $\log K_{\mathrm{HSA}}$ (range for $95 \%$ of drugs is -1.5 to 1.2) [41]; the logarithm of predicted blood/brain barrier partition coefficient, $\log B / B$ (range for $95 \%$ of drugs is -3.0 to 1.0 ) [42-44]; the predicted apparent Caco-2 cell membrane permeability $\left(\mathrm{BI} P_{\text {Caco-2}}\right)$ in Boehringer-Ingelheim scale, in $\mathrm{nm} / \mathrm{s}$ (range for $95 \%$ of drugs is $<5$ low, $>100$ high) [45-47]; the predicted apparent Madin-Darby canine kidney (MDCK) cell permeability in $\mathrm{nm} \mathrm{s}^{-1}(<25$ poor, $>500$ great) [46]; the index of cohesion interaction in solids, Ind $_{\text {coh }}$, calculated from the number of hydrogen bond acceptors (HBA), hydrogen bond donors (HBD) and the surface area accessible to the solvent $\left(S_{\mathrm{mol}}\right)$ by the relation Ind $_{\text {coh }}=\mathrm{HBA} \times \sqrt{\mathrm{HBD}} / S_{\text {mol }} \quad(0.0$ to 0.05 for $95 \%$ of drugs) [40]; the globularity descriptor, Glob $=\left(4 \pi r^{2}\right) / S_{\mathrm{mol}}$, where $r$ is the radius of the sphere whose volume is equal to the molecular volume ( 0.75 to 0.95 for $95 \%$ of drugs); the predicted polarizability, $\mathrm{QP}_{\text {polrz }}(13.0$ to 70.0 for $95 \%$ of drugs); the predicted $\mathrm{IC}_{50}$ value for blockage of HERG $\mathrm{K}^{+}$channels, logHERG (concern <-5) [48,49]; the predicted skin permeability, $\log K_{\mathrm{p}}$ ( -8.0 to -1.0 for $95 \%$ of drugs) [50,51]; and the number of likely metabolic reactions, \#metab (range for $95 \%$ of drugs is 0 to 15 ).

\section{Results and discussion}

\section{Overall DMPK compliance of the CamMedNP library}

The 24 most relevant molecular descriptors calculated by QikProp are used to determine the \#star parameter [52]. A plot of the \#stars parameter (on the $x$-axis) against the corresponding counts (on the $y$-axis) in the CamMedNP is shown within the same set of axes with those of the 'drug-like', 'lead-like' and 'fragment-like' standard subsets, Figure 1. The criteria for the respective standard subsets were defined as $\mathrm{MW}<500, \log P<5, \mathrm{HBD} \leq 5, \mathrm{HBA} \leq$ 10 [14]; $150 \leq \mathrm{MW} \leq 350, \log P \leq 4, \mathrm{HBD} \leq 3, \mathrm{HBA} \leq 6$ [53-55] and MW $\leq 250,-2 \leq \log P \leq 3, \mathrm{HBD}<3$, HBA $<$ 6 , NRB $<3$ [56]. QikProp was unable to compute the ADMET descriptors for 25 compounds out of the total library due to limitations that were not clear to us. Of the remaining 1,834 compounds, $48.04 \%$ showed \#star $=0$, while $74.21 \%$ had \#star $\leq 2$. Among the 1,122 compounds of the drug-like subset, $79.12 \%$ had pharmacokinetic descriptors within the acceptable range for $95 \%$ of known drugs, while $97.33 \%$ showed \#stars $\leq 2$. The lead-like and fragment-like subsets were, respectively, $81.15 \%$ and $55.56 \%$ compliant for all of the 24 most relevant computed descriptors. The mean values for 19 selected computed descriptors have been shown in Table 1 for all four compound libraries, while the percentage compliances for 14 selected ADMET-related descriptors are shown in

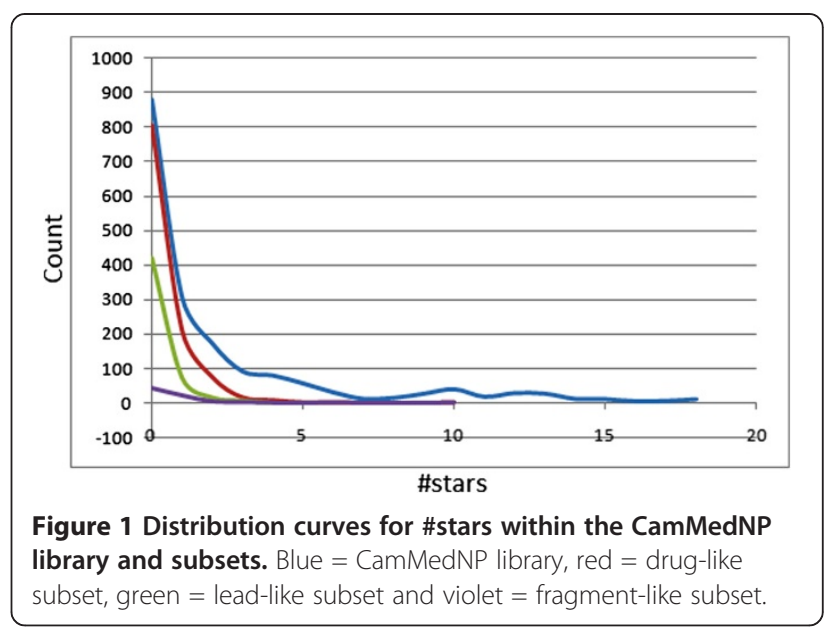

Table 2. The mean values and percentage compliances indicate a high probability of finding drug leads within the CamMedNP compound library.

\section{Bioavailability prediction}

The bioavailability of a compound depends on the processes of absorption and liver first-pass metabolism [57]. The absorption, in turn, depends on the solubility and permeability of the compound, as well as on the interactions with transporters and metabolizing enzymes in the gut wall. The computed parameters used to assess oral absorption are the predicted aqueous solubility, $\log S_{\text {wat }}$, the conformation-independent predicted aqueous solubility, CI $\log S_{\text {wat }}$, the predicted qualitative human oral absorption, the predicted \% human oral absorption and compliance to Jorgensen's famous 'Rule of Three' (ro3). The solubility calculation procedure implemented depends on the similarity property space between the given molecule and its most similar analogue within the experimental training set used to develop the model implemented in QikProp, i.e. if the similarity is $<0.9$, then the QikProp predicted value is taken; otherwise, the predicted property, $P_{\text {pred }}$, is adjusted such that

$$
P_{\text {pred }}=S P_{\exp }+(1-S) P_{Q P}
$$

where $S$ is the similarity and $P_{\exp }$ and $P_{\mathrm{QP}}$ are, respectively, the experimental and QikProp predictions for the most similar molecule within the training set. In Equation 1, if $S=1$, then the predicted property is equal to the measured experimental property of the training set compound. According to Jorgensen's ro3, if a compound complies to all or some of the rules $\left(\log S_{\text {wat }}>-5.7\right.$, $\mathrm{BI} P_{\text {Caco-2 }}>22 \mathrm{~nm} / \mathrm{s}$ and number of primary metabolites $<$ seven), then it is more likely to be orally available. The distribution curves for two of the three determinants for the ro3 $\left(\log S_{\text {wat }}\right.$ and $\left.\mathrm{BI} P_{\mathrm{Caco-}-2}\right)$ are shown in Figure $2 \mathrm{~A}, \mathrm{~B}$. In general, $47.22 \%$ of the CamMedNP library was compliant 
Table 1 Average pharmacokinetic property distributions of total CamMedNP library in comparison with various subsets

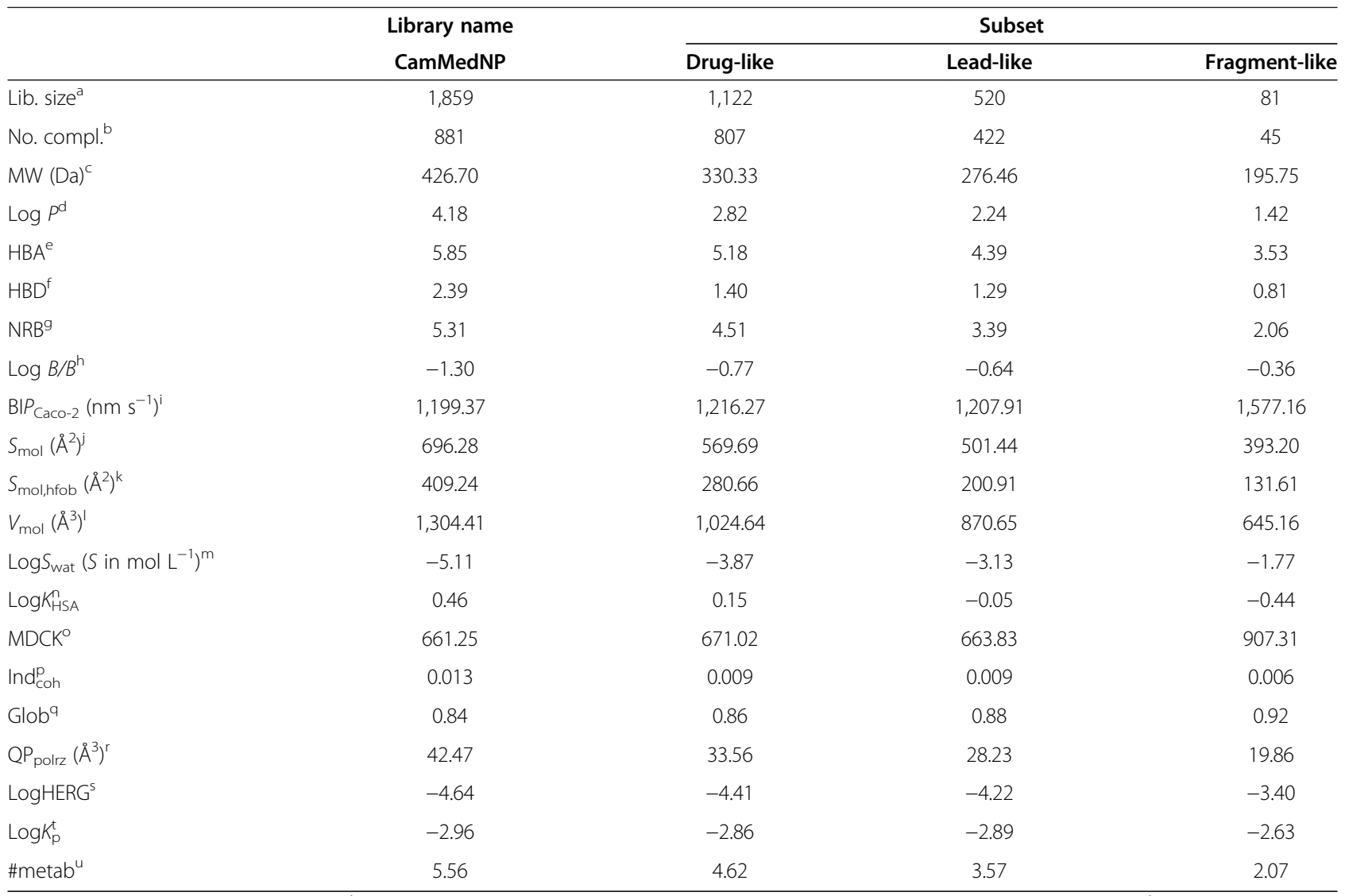

${ }^{a}$ Size or number of compounds in library; ${ }^{b}$ number of compounds with \#star $=0 ;{ }^{c}$ molar weight (range for $95 \%$ of drugs is 130 to 725 Da); ${ }^{d}$ logarithm of partitioning coefficient between $n$-octanol and water phases (range for $95 \%$ of drugs is -2 to 6 ); ${ }^{e}$ number of hydrogen bonds accepted by the molecule (range for $95 \%$ of drugs is 2 to 20 ); ${ }^{f}$ number of hydrogen bonds donated by the molecule (range for $95 \%$ of drugs is 0 to 6 ); ${ }^{9}$ number of rotatable bonds (range for $95 \%$ of drugs is 0 to 15); ${ }^{h}$ logarithm of predicted blood/brain barrier partition coefficient (range for $95 \%$ of drugs is -3.0 to 1.0 ); i predicted apparent Caco- 2 cell membrane permeability in Boehringer-Ingelheim scale, in $\mathrm{nm} / \mathrm{s}$ (range for $95 \%$ of drugs is $<5$ low, $>100$ high); ${ }^{j}$ total solvent-accessible molecular surface, in $\AA^{2}$ (probe radius $1.4 \AA$; range for $95 \%$ of drugs is 300 to $1,000 \AA^{2}$ ); khydrophobic portion of the solvent-accessible molecular surface, in $\AA^{2}$ (probe radius $1.4 \AA^{\text {; }}$ range for $95 \%$ of drugs is 0 to $750 \AA^{2}$ ); 'total volume of molecule enclosed by solvent-accessible molecular surface, in $\AA^{3}$ (probe radius $1.4 \AA$; range for $95 \%$ of drugs is 500 to $2,000 \AA^{3}$ ); mlogarithm of aqueous solubility in $\mathrm{g} / \mathrm{dm}^{3}$ (range for $95 \%$ of drugs is -6.0 to 0.5 ); ${ }^{\mathrm{n}}$ logarithm of predicted binding constant to human serum albumin (range for $95 \%$ of drugs is -1.5

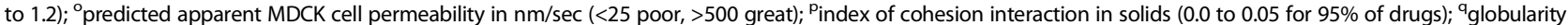

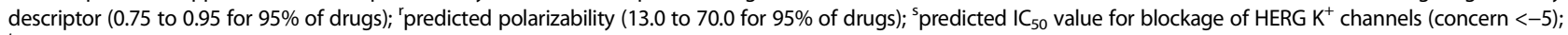

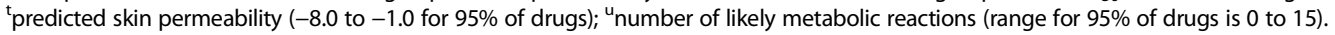

to the ro3, while the respective percentage compliances for the various subsets were $72.28 \%, 92.11 \%$ and $100 \%$ for the drug-like, lead-like and fragment-like libraries. Among the individual computed parameters, the most remarkable was $\log S_{\text {wat }}$, which was met by $75.74 \%$ of the compounds within the CamMedNP library, while this property shows a Gaussian distribution for the drug-like and lead-like subsets. Only $37.94 \%$ of the compounds fell within the respected range for the $\mathrm{BI} P_{\text {Caco-2 }}$ criterion. The predicted apparent Caco- 2 cell permeability, $\mathrm{BI} P_{\text {Caco-2 }}\left(\right.$ in $\mathrm{nm} \mathrm{s}^{-1}$ ), models the permeability of the gut-blood barrier (for nonactive transport), even though this parameter is not often correctly predicted computationally [58]. The histograms of the predicted qualitative human oral absorption parameter (in the scale 1 = low, $2=$ medium and $3=$ high) are shown in Figure 3. It was observed that $52.45 \%$ of the compounds in CamMedNP were predicted to have high human oral absorption. The predicted \% human oral absorption (on 0 to $100 \%$ scale) shows a similar trend, with $41.06 \%$ of the compounds being predicted to be absorbed at $100 \%$, and $57.96 \%$ of the compounds predicted to be absorbed at $>90 \%$.

The size of a molecule, as well as its capacity to make hydrogen bonds, its overall lipophilicity, its shape and flexibility are important properties to consider when determining permeability. Molecular flexibility has been seen as a parameter which is dependent on the number of rotatable bonds (NRB), a property which influences the bioavailability in rats [58]. The distribution of the NRB for this dataset has been previously discussed [29] and revealed that the compounds within the CamMedNP library show some degree of conformational flexibility, the peak 
Table 2 Percentage compliances of selected ADMET-related descriptors of total CamMedNP library in comparison with various subsets

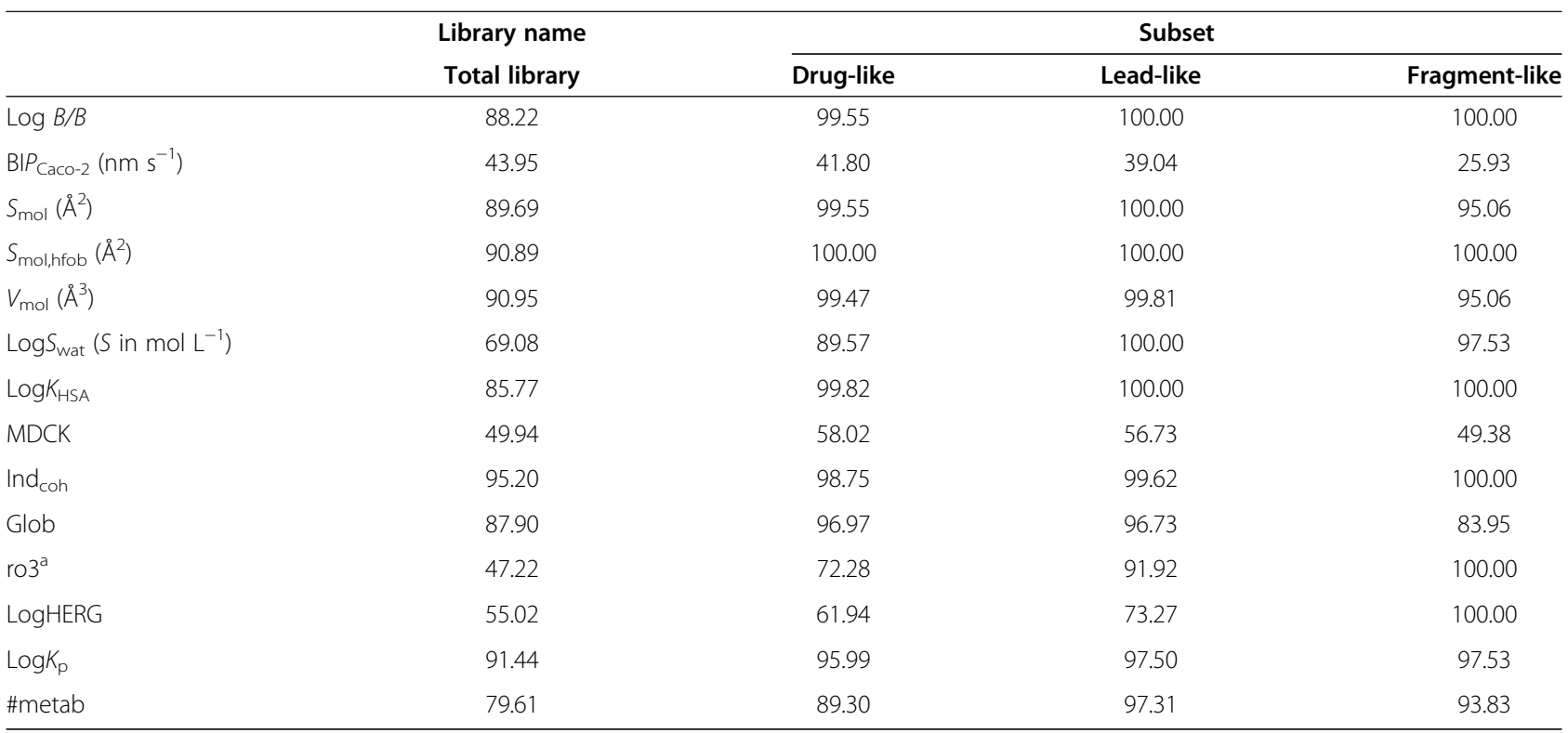

The descriptors of the entries in the first column are defined in Table $1 ;$ a percentage compliance to Jorgensen's Rule of Three.

value for the NRB being between 1 and 2, while the average value is 5.31 (Table 1 ).

\section{Prediction of blood-brain barrier penetration}

Too polar drugs do not cross the BBB. The blood/brain partition coefficients $(\log B / B)$ were computed and used as a predictor for access to the central nervous system (CNS). The predicted CNS activity was computed on a -2 (inactive) to +2 (active) scale and showed that only $1.85 \%$ of the compounds in the CamMedNP could be active in the CNS (predicted CNS activity $>1$ ). A distribution of the log $B / B$ (Figure 4) shows a right-slanted Gaussian-shaped curve with a peak at $-0.5 \log B / B$ units (the same for all the standard subsets), with $>88 \%$ of the compounds in the
CamMedNP falling within the recommended range for the predicted brain/blood partition coefficient (-3.0 to 1.2). The MDCK monolayers are widely used to make oral absorption estimates, the reason being that these cells also express transporter proteins, but only express very low levels of metabolizing enzymes [58]. They are also used as an additional criterion to predict BBB penetration. Thus, our calculated apparent MDCK cell permeability could be considered to be a good mimic for the BBB (for non-active transport). It was estimated that only about $50 \%$ of the compounds had apparent MDCK cell permeabilities falling within the recommended range of 25 to $500 \mathrm{~nm} \mathrm{~s}^{-1}$ for $95 \%$ of known drugs. This situation was not greatly improved in the druglike and lead-like subsets (58\% and 57\%, respectively).
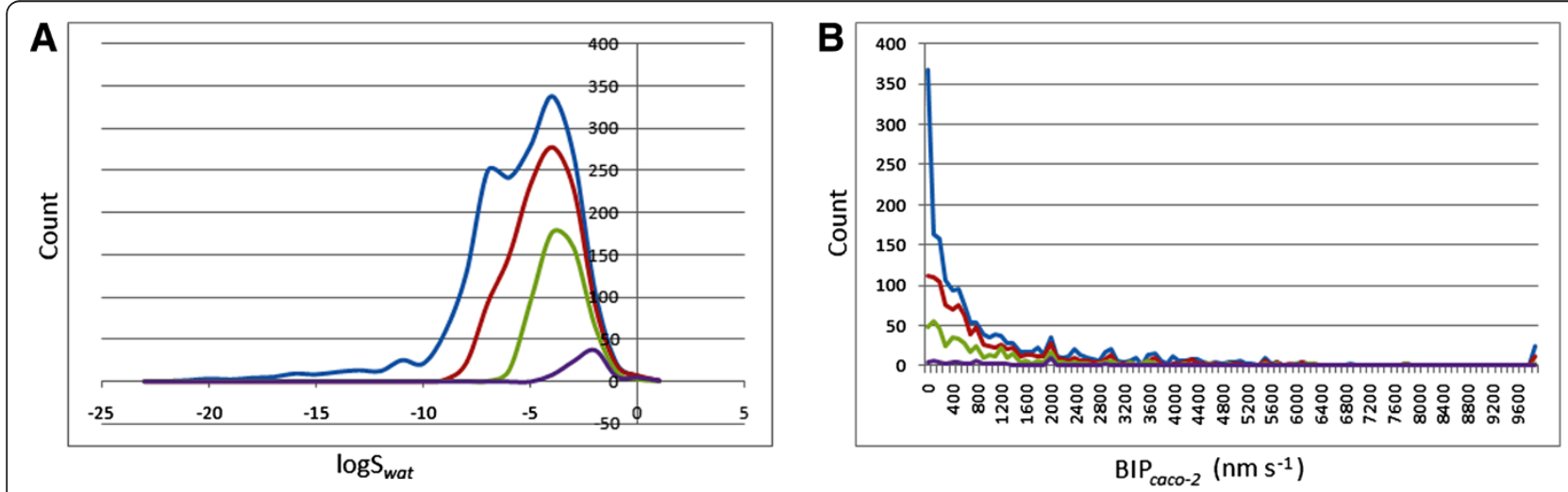

Figure 2 Distribution curves for compliance to Jorgensen's 'Rule of Three'. (A) calculated log $S_{\text {wat }}$ against count. (B) Predicted BIP caco-2 against count. Blue $=$ CamMedNP library, red $=$ drug-like subset, green $=$ lead-like subset and violet $=$ fragment-like subset. 


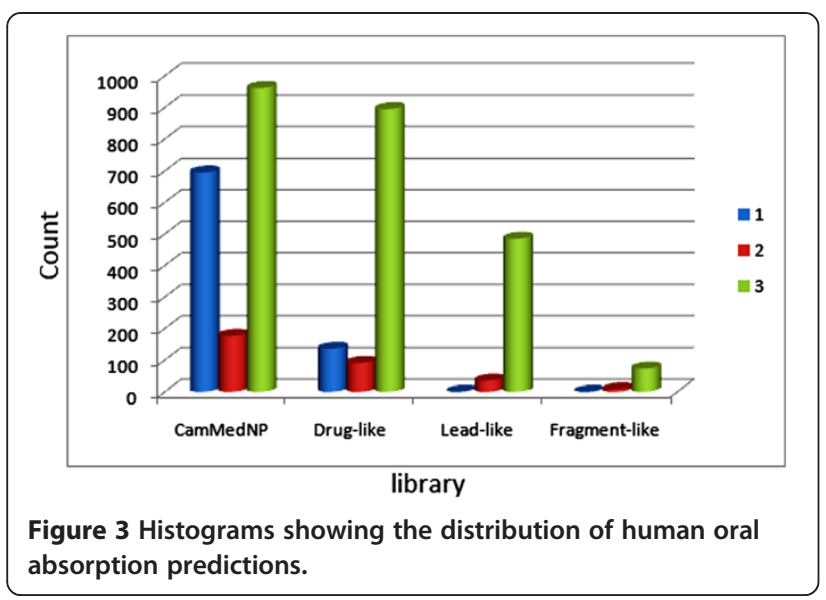

\section{Prediction of dermal penetration}

This factor is important for drugs administered through the skin. The distribution of computed skin permeability parameter, $\log K_{\mathrm{p}}$, showed smooth Gaussian-shaped graphs centred at $-2.5 \log K_{\mathrm{p}}$ units for all the four datasets (Figure 5), with approximately $91 \%$ of the compounds in the CamMedNP database falling within the recommended range for $>95 \%$ of known drugs. The predicted maximum transdermal transport rates, $J_{\mathrm{m}}$ (in $\mu \mathrm{cm}^{-2} \mathrm{~h}^{-1}$ ), were computed from the aqueous solubility $\left(S_{\text {wat }}\right)$, the MW and skin permeability $\left(K_{\mathrm{p}}\right)$ using the relation $(2)$ :

$$
J_{\mathrm{m}}=K_{\mathrm{p}} \times \mathrm{MW} \times \mathrm{S}_{\mathrm{wat}}
$$

This parameter showed variations from 0 to $1,603 \mu$ $\mathrm{cm}^{-2} \mathrm{~h}^{-1}$, with only about $1.39 \%$ of the compounds in CamMedNP having the predicted value of $J_{\mathrm{m}}>100 \mu$ $\mathrm{cm}^{-2} \mathrm{~h}^{-1}$.

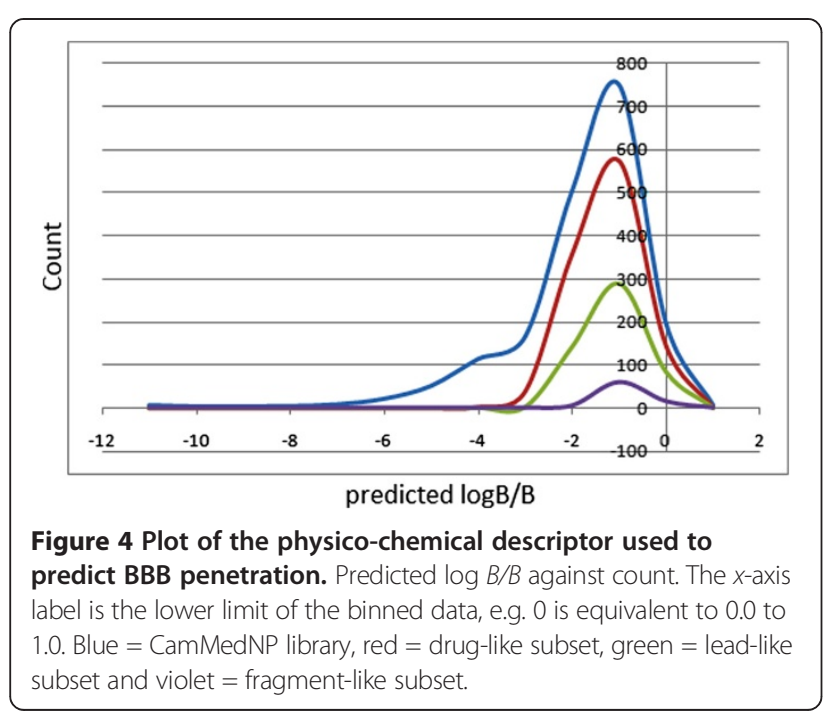

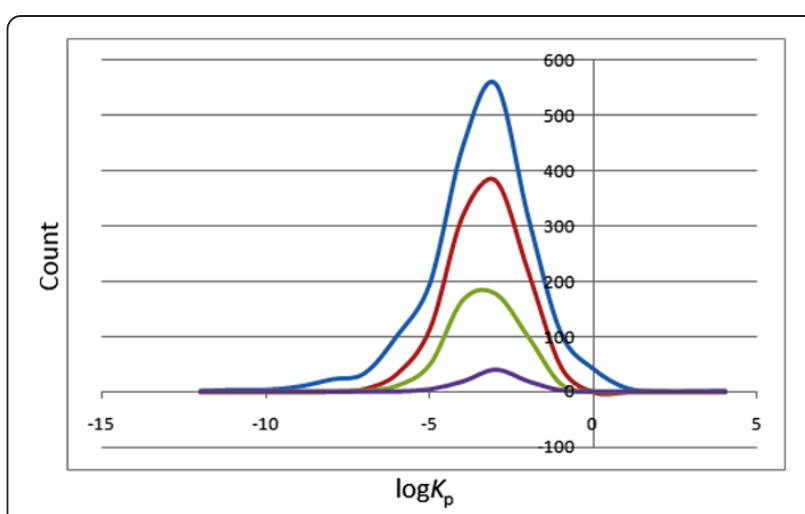

Figure 5 Distribution curves for the predicted skin penetration parameter. Blue $=$ CamMedNP library, red $=$ drug-like subset, green $=$ lead-like subset and violet $=$ fragment-like subset.

\section{Prediction of plasma-protein binding}

The efficiency of a drug may be affected by the degree to which it binds to the proteins within the blood plasma. It is noteworthy that the binding of drugs to the plasma proteins (like human serum albumin, lipoprotein, glycoprotein, $\alpha, \beta$ and $\gamma$ globulins) greatly reduces the quantity of the drug in the general blood circulation, and hence, the less bound a drug is, the more efficiently it can traverse cell membranes or diffuse. The predicted plasma-protein binding has been estimated by the prediction of binding to human serum albumin; the $\log K_{\mathrm{HSA}}$ parameter recommended range is -1.5 to 1.5 for $95 \%$ of known drugs. Figure 6 shows the variation of this calculated parameter within the CamMedNP dataset, as well as for the standard subsets. This equally gave smooth Gaussian-shaped curves centred on $-0.5 \log K_{\mathrm{HSA}}$ units for all the four datasets. In addition, our calculations revealed that $>85 \%$ of the compounds within the CamMedNP library are compliant to this parameter, indicating that a majority of the compounds are likely to circulate freely within the blood stream and, hence, have access to the target site.

\section{Metabolism prediction}

An estimated number of possible metabolic reactions has also been predicted by QikProp and used to determine whether the molecules can easily gain access to the target site after entering the blood stream. The average estimated number of possible metabolic reactions for the CamMedNP library was between five and six, while those of the standard subsets drop sequentially by one step in a progressive manner (Table 1). Even though some of the compounds are likely to undergo as many as up to 26 metabolic reactions due to the complexity of some of the plant secondary metabolites within the database (Figure 7), about $80 \%$ of the compounds are predicted to undergo the recommended number of metabolic steps (one to eight reactions), with the situation improving to around $90 \%$ and approximately 


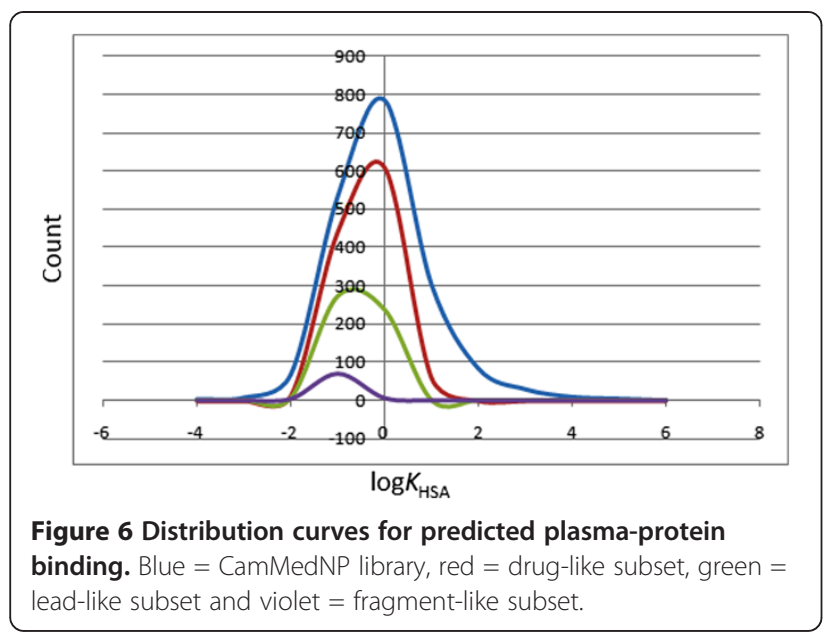

97\% in the drug-like and lead-like subsets, respectively. From Figure 7, it can be observed that, except for the fragment-like subsets which peaks at two predicted metabolic reactions, the peak values for the number of predicted metabolic reactions were at three for all of the datasets.

\section{Prediction of blockage of human ether-a-go-go-related gene potassium channel}

Human ether-a-go-go-related gene (HERG) encodes a potassium ion $\left(\mathrm{K}^{+}\right)$channel that is implicated in the fatal arrhythmia known as torsade de pointes or the long QT syndrome [59]. The HERG $\mathrm{K}^{+}$channel, which is best known for its contribution to the electrical activity of the heart which coordinates the heart's beating, appears to be the molecular target responsible for the cardiac toxicity of a wide range of therapeutic drugs [60]. HERG has also been associated with modulating the functions of some cells of the nervous system and with establishing and maintaining cancer-like features in leukemic cells [61]. Thus, HERG $\mathrm{K}^{+}$channel blockers are potentially toxic, and the predicted $\mathrm{IC}_{50}$ values often provide reasonable

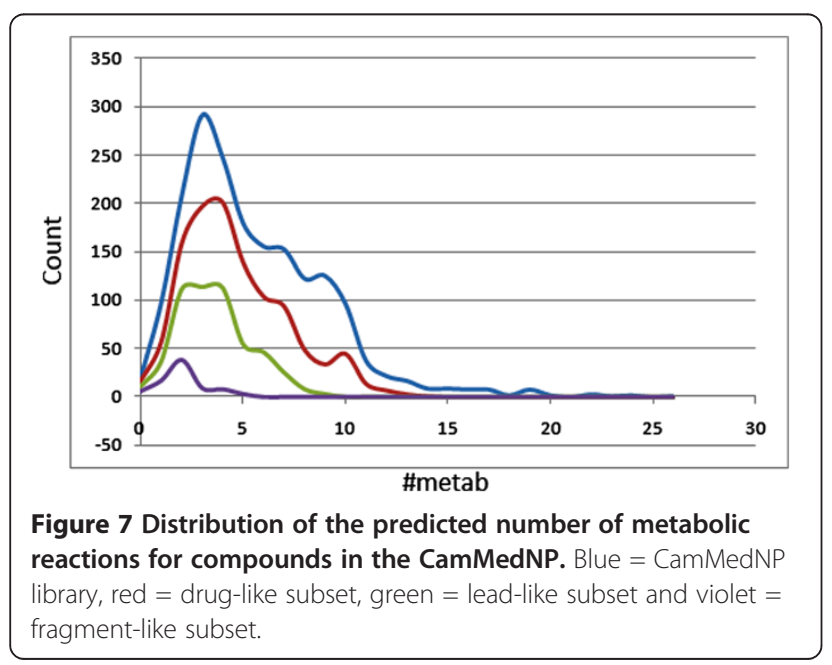

predictions for cardiac toxicity of drugs in the early stages of drug discovery [62]. In this work, the estimated or predicted $\mathrm{IC}_{50}$ values for blockage of this channel have been used to model the process in silico. The recommended range for the predicted $\log \mathrm{IC}_{50}$ values for blockage of the HERG $\mathrm{K}^{+}$channels $(\operatorname{logHERG})$ is $>-5$. A distribution curve for the variation of the predicted $\operatorname{logHERG}$ is shown in Figure 8, which is left-slanted Gaussian-shaped curve with a peak at $-5.5 \operatorname{logHERG}$ units for both the total library and the drug-like subset, meanwhile the lead-like library rather peaks at -4.5 units. It was observed that, in general, this parameter is predicted to fall within the recommended range for about $55 \%$ of the compounds within the CamMedNP database, approximately $62 \%$ for the drug-like subset and around $73 \%$ for the lead-like subset.

\section{Usefulness of the CamMedNP library}

The usefulness of the CamMedNP database in lead generation has been exemplified with the docking and pharmacophore-based screening for potential inhibitors of a validated anti-malarial drug target in our laboratory, and the results will be published in a subsequent paper. It is important to mention that virtual screening results could provide insight and direct natural products chemists to search for theoretically active principles with attractive ADMET profiles, which have been previously isolated, but not tested for activity against specified drug targets (if samples are absent). This 'resurrection' process could prove to be a better procedure for lead search than the random screening, which is a common practice in our Cameroonian laboratories. CamMedNP is constantly being updated; meanwhile, a MySQL platform (Cupertino, USA) to facilitate the searching of this database and ordering of compound samples is under development within our group and will also be published subsequently. However, 3D structures of the compounds, as well as their physico-chemical properties that were used to evaluate the DMPK profile, can be

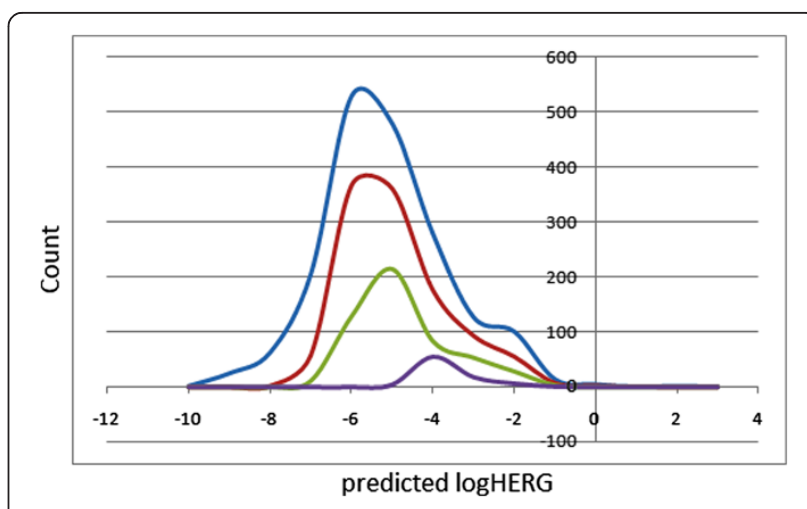

Figure $8 \mathrm{~A}$ plot of the predicted logHERG values for the CamMedNP and standard subsets. Blue $=$ CamMedNP library, red = drug-like subset, green $=$ lead-like subset and violet $=$ fragment-like subset. 
freely downloaded as additional files accompanying this publication (see Additional file 1, Additional file 2, Additional file 3, Additional file 4). In addition, information about compound sample availability can be obtained on request from the authors of this paper or from the pan-African Natural Products Library (p-ANAPL) project $[63,64]$.

\section{Conclusion}

Modern drug discovery programs usually involve the search for small molecule leads with attractive pharmacokinetic profiles. The presence of such within the CamMedNP library is of major importance and, therefore, renders the database attractive, in addition to the alreadyknown properties (drug-like, lead-like fragment-like and diverse). This is an indication that the 3D structures of naturally occurring compounds within the CamMedNP could be a good starting point for docking, neural networking and pharmacophore-based virtual screening campaigns, thus rendering the CamMedNP as a useful asset for the drug discovery community. 3D structures of the compounds, as well as their physico-chemical properties that were used to evaluate the DMPK profile of the CamMedNP library, can be freely downloaded (for noncommercial use) as additional files which accompany this publication (see Additional file 1, Additional file 2, Additional file 3, Additional file 4). The physical samples for testing are available at the various research laboratories in Cameroon in varying quantities. Questions regarding the availability of the compound samples could be addressed directly to the authors of this paper. Otherwise, the samples could be obtainable from the p-ANAPL consortium, which has a mandate to collect samples of NPs from the entire continent of Africa and make them available for biological screening. This network is being set up under the auspices of the Network for Analytical and Bioassay Services in Africa [63,64].

\section{Additional files}

\section{Additional file 1: Compounds currently included in CamMedNP.}

$13 \mathrm{D}$ structures of compounds currently included in CamMedNP with calculated pharmacokinetic descriptors.

Additional file 2: Drug-like subset. 3D structures of the drug-like subset with calculated pharmacokinetic descriptors.

Additional file 3: Lead-like subset. 3D structures of the lead-like subset with calculated pharmacokinetic descriptors.

Additional file 4: Fragment-like subset. 3D structures of the fragment-like subset with calculated pharmacokinetic descriptors.

\section{Abbreviations}

3D: Three dimensional; ADME/T: Absorption distribution, metabolism, excretion and toxicity; CamMedNP: Cameroonian medicinal plant and natural products database; DMPK: Drug metabolism and pharmacokinetics; $\log P$ : logarithm of the octan-1-ol/water partition coefficient; MDCK: Madin-
Darby canine kidney; MW: Molar weight; NP: Natural product; NRB: Number of rotatable bonds; p-ANAPL: pan-African natural products library.

\section{Competing interests}

The authors declare that they have no competing interests.

\section{Authors' contributions}

WS, EM, LCOO and SMNE conceived the project. All authors participated in the data generation and analysis, discussion of results and the conception of the paper. FNK wrote the first draft of the paper. This work is part of the PhD project of FNK. All authors read and approved the final manuscript.

\section{Authors' information}

WS and SMNE are professors of Medicinal Chemistry with an interest in CADD, while SMNE also focuses on organic synthesis and on natural product leads from the Cameroonian medicinal plants. LMM and JAM are natural products chemists actively involved in the isolation and characterization of secondary metabolites from the Cameroonian medicinal plants. LLL holds a $\mathrm{PhD}$ in Environmental Science and manages the Chemical and Bioactivity Information Centre (CBIC) with a focus on developing databases for information from medicinal herbs in Africa. PNJ is a retired research officer of Lhasa Ltd., who currently leads the CBIC branch in Leeds, UK. FNK is a PhD student working on CADD under the joint supervision of LCOO and EM.

\section{Acknowledgements}

Financial support is acknowledged from the German Academic Exchange Service (DAAD) to FNK for his stay in Halle, Germany for part of his PhD and from the International Centre for Theoretical Physics (ICTP), Trieste, Italy, via the OEA-AC71 Program. The authors also acknowledge the academic licence generously offered by Schrodinger Inc., for this work. The assistance of Mr. Vincent de Paul Nzuwah Nziko (graduate student, Chemistry and Biochemistry, Utah State University, USA) is also acknowledged for proofreading the manuscript.

\section{Author details}

'CEPAMOQ, Faculty of Science, University of Douala, P.O. Box 8580, Douala, Cameroon. ${ }^{2}$ Chemical and Bioactivity Information Centre, Department of Chemistry, Faculty of Science, University of Buea, P.O. Box 63, Buea, Cameroon. ${ }^{3}$ Department of Pharmaceutical Sciences, Martin Luther University of Halle-Wittenberg, Wolfgang-Langenbeck Str. 4, Halle (Saale) 06120, Germany. ${ }^{4}$ Department of Chemistry, Faculty of Science, University of Buea, P.O. Box 63, Buea, Cameroon. ${ }^{5}$ Laboratory for Simulations and Biomolecular Physics, Advanced Teachers Training College, University of Yaoundé I, P.O. Box 47, Yaoundé, Cameroon. 'Laboratory of Fundamental and Applied Physics, University of Abobo-Adjame, Abidjan 02 BP 801, Cote d'Ivoire, Africa. ${ }^{7}$ Department of Chemistry, Faculty of Science, University of Douala, P.O. Box 24157, Douala, Cameroon. ${ }^{8}$ Chemical and Bioactivity Information Centre, 22-23 Blenheim Terrace, Woodhouse Lane, Leeds LS2 9HD, UK.

Received: 2 July 2013 Accepted: 15 August 2013

Published: 30 August 2013

\section{References}

1. Li JWH, Vederas JC (2009) Drug discovery and natural products: end of an era or an endless frontier? Science 325:161-165

2. Chin YW, Balunas MJ, Chai HB, Kinghorn AD (2006) Drug discovery from natural sources. AAPS J 8(2):E239-E253

3. Newman DJ (2008) Natural products as leads to potential drugs: an old process or the new hope for drug discovery? J Med Chem 51:2589-2599

4. Harvey AL (2008) Natural products in drug discovery. Drug Discov Today 13:894-901

5. Koehn FE, Carter GT (2005) The evolving role of natural products in drug discovery. Nat Rev Drug Discov 4:206-220

6. Efange SMN (2002) Natural products: a continuing source of inspiration for the medicinal chemist. In: Iwu MM, Wootton JC (eds) Advances in phytomedicine. Elsevier, Amsterdam

7. Butler MS (2005) Natural products to drugs: natural product derived compounds in clinical trials. Nat Prod Rep 22:162-195

8. Wetzel S, Schuffenhauer A, Roggo S, Ertl P, Waldmann H (2007) Cheminformatic analysis of natural products and their chemical space. Chimia Int J Chem 61:355-360 
9. Grabowski K, Baringhaus K-H, Schneider G (2008) Scaffold diversity of natural products: inspiration for combinatorial library design. Nat Prod Rep 25:892-904

10. Grabowski K, Schneider G (2007) Properties and architecture of drugs and natural products revisited. Curr Chem Biol 1:115-127

11. Quinn RJ, Carroll AR, Pham MB, Baron P, Palframan ME, Suraweera L, Pierens GK, Muresan S (2008) Developing a drug-like natural product library. J Nat Prod 71:464-468

12. Hodgson J (2001) ADMET - turning chemicals into drugs. Nat Biotechnol 19:722-726

13. Navia MA, Chaturvedi PR (1996) Design principles for orally bioavailable drugs. Drug Dev Today 1:179-189

14. Lipinski CA, Lombardo F, Dominy BW, Feeney PJ (1997) Experimental and computational approaches to estimate solubility and permeability in drug discovery and development settings. Adv Drug Delivery Rev 23:3-25

15. Lombardo F, Gifford E, Shalaeva MY (2003) In silico ADME prediction: data, models, facts and myths. Mini Rev Med Chem 3:861-875

16. Gleeson MP, Hersey A, Hannongbua S (2011) In-silico ADME models: a general assessment of their utility in drug discovery applications. Curr Top Med Chem 11(4):358-381

17. DiMasi JA, Hansen RW, Grabowsk HG (2003) The price of innovation: new estimates of drug development costs. J Health Econ 22:151-185

18. Darvas F, Keseru G, Papp A, Dormán G, Urge L, Krajcsi P (2002) In silico and ex silico ADME approaches for drug discovery. Top Med Chem 2:1287-1304

19. eADMET (2011) OCHEM - our platform for the creation of in silico ADME/tox prediction models http://www.eadmet.com/en/ochem.php. Accessed 21 June 2013

20. Lhasa Ltd (2010) Meteor, version 13.0.0. Lhasa Ltd, Leeds, UK

21. Schrödinger (2011) QikProp, version 3.4. LLC, New York, NY

22. Cruciani C, Crivori P, Carrupt PA, Testa B (2000) Molecular fields in quantitative structure-permeation relationships: the VolSurf approach. J Mol Struc-Theochem 503:17-30

23. Tetko IV, Bruneau P, Mewes H-W, Rohrer DC, Poda Gl (2006) Can we estimate the accuracy of ADMET predictions? Drug Discov Today 11:700-707

24. Hansch C, Leo A, Mekapatia SB, Kurup A (2004) QSAR and ADME. Bioorg Med Chem 12:3391-3400

25. Greene N, Judson PN, Langowski JJ (1999) Knowledge-based expert systems for toxicity and metabolism prediction: DEREK, StAR and METEOR. SAR QSAR Environ Res 10:299-314

26. Button WG, Judson PN, Long A, Vessey JD (2003) Using absolute and relative reasoning in the prediction of the potential metabolism of xenobiotics. J Chem Inf Comput Sci 43:1371-1377

27. Cronin MTD (2003) Computer-assisted prediction of drug toxicity and metabolism in modern methods of drug discovery. In: Hilgenfeld R, Hillisch A (eds) Modern methods of drug discovery. Birkhäuser, Basel

28. Hou T, Wang J (2008) Structure-ADME relationship: still a long way to go? Expert Opin Drug Metab Toxicol 4(6):759-770

29. Ntie-Kang F, Mbah JA, Mbaze LM, Lifongo LL, Scharfe M, Ngo Hanna J, ChoNgwa F, Amoa Onguéné P, Owono Owono LC, Megnassan E, Sippl W, Efange SMN (2013) CamMedNP: building the Cameroonian 3D structural natural products database for virtual screening. BMC Complement Altern Med 13:88

30. Ntie-Kang F, Lifongo LL, Mbaze LM, Ekwelle N, Owono Owono LC, Megnassan E, Judson PN, Sippl W, Efange SMN (2013) Cameroonian medicinal plants: a bioactivity versus ethnobotanical survey and chemotaxonomic classification. BMC Complement Altern Med 13:147

31. Chemical Computing Group, Inc (2010) Molecular operating environment software. CCG, Montreal

32. Halgren TA (1996) Merck molecular force field. J Comput Chem 17:490-641

33. Schrödinger (2011) LigPrep software, version 2.5. LLC, New York, NY

34. Schrödinger (2011) Maestro, version 9.2. LLC, New York, NY

35. Shivakumar D, Williams J, Wu Y, Damm W, Shelley J, Sherman W (2010) Prediction of absolute solvation free energies using molecular dynamics free energy perturbation and the OPLS force field. J Chem Theory Comput 6:1509-1519

36. Jorgensen WL, Maxwell DS, Tirado-Rives J (1996) Development and testing of the OPLS all-atom force field on conformational energetics and properties of organic liquids. J Am Chem Soc 118(45):11225-11236

37. Jorgensen WL, Tirado-Rives J (1988) The OPLS (optimized potentials for liquid simulations) potential functions for proteins, energy minimizations for crystals of cyclic peptides and crambin. J Am Chem Soc 110(6):1657-1666
38. Jorgensen WL, Duffy EM (2002) Prediction of drug solubility from structure. Adv Drug Deliv Rev 54:355-366

39. Duffy EM, Jorgensen WL (2000) Prediction of properties from simulations: free energies of solvation in hexadecane, octanol, and water. J Am Chem Soc 122:2878-2888

40. Jorgensen WL, Duffy EM (2000) Prediction of drug solubility from Monte Carlo simulations. Bioorg Med Chem Lett 10:1155-1158

41. Colmenarejo G, Alvarez-Pedraglio A, Lavandera J-L (2001) Cheminformatic models to predict binding affinities to human serum albumin. J Med Chem 44:4370-4378

42. Luco JM (1999) Prediction of brain-blood distribution of a large set of drugs from structurally derived descriptors using partial least squares (PLS) modelling. J Chem Inf Comput Sci 39:396-404

43. Kelder J, Grootenhuis PD, Bayada DM, Delbresine LP, Ploemen JP (1999) Polar molecular surface as a dominating determinant for oral absorption and brain penetration of drugs. Pharm Res 16:1514-1519

44. Ajay BGW, Murkco MA (1999) Designing libraries with CNS activity. J Med Chem 42:4942-4951

45. Yazdanian M, Glynn SL, Wright JL, Hawi A (1998) Correlating partitioning and Caco-2 cell permeability of structurally diverse small molecular weight compounds. Pharm Res 15:1490-1494

46. Irvine JD, Takahashi L, Lockhart K, Cheong J, Tolan JW, Selick HE, Grove JR (1999) MDCK (Madin-Darby canine kidney) cells: a tool for membrane permeability screening. J Pharm Sci 88:28-33

47. Stenberg P, Norinder U, Luthman K, Artursson P (2001) Experimental and computational screening models for the prediction of intestinal drug absorption. J Med Chem 44:1927-1937

48. Cavalli A, Poluzzi E, De Ponti F, Recanatini M (2002) Toward a pharmacophore for drugs inducing the long QT syndrome: insights from a COMFA Study of HERG K+ channel blockers. J Med Chem 45:3844-3853

49. De Ponti F, Poluzzi E, Montanaro N (2001) Organising evidence on QT prolongation and occurrence of Torsades de Pointes with nonantiarrhythmic drugs: a call for consensus. Eur J Clin Pharmacol 57:185-209

50. Potts RO, Guy RH (1992) Skin permeability. Pharm Res 9:663-669

51. Potts RO, Guy RH (1995) A predictive algorithm for skin permeability: the effects of molecular size and hydrogen bond activity. Pharm Res 12:1628-1633

52. Schrödinger Press (2011) QikProp 3.4 user manual. LLC, New York, NY

53. Teague SJ, Davis AM, Leeson PD, Opea TI (1999) The design of leadlike combinatorial libraries. Angew Chem Int Ed 38:3743-3748

54. Oprea TI (2002) Current trends in lead discovery: are we looking for the appropriate properties? J Comput-Aided Mol Des 16:325-334

55. Schneider G (2002) Trends in virtual computational library design. Curr Med Chem 9:2095-2102

56. Verdonk ML, Cole JC, Hartshorn ML, Murray CW, Taylor RD (2003) Improved protein-ligand docking using GOLD. Proteins 52:609-623

57. Van de Waterbeemd H, Gifford E (2003) ADMET in silico modelling: towards prediction paradise? Nat Rev Drug Discov 2:192-204

58. Veber DF, Johnson SR, Cheng HY, Smith BR, Ward KW, Kopple KD (2002) Molecular properties that influence the oral bioavailability of drug candidates. J Med Chem 45:2615-2623

59. Hedley PL, Jørgensen P, Schlamowitz S, Wangari R, Moolman-Smook J, Brink PA, Kanters JK, Corfield VA, Christiansen M (2009) The genetic basis of long QT and short QT syndromes: a mutation update. Hum Mutat 30:1486-1511

60. Vandenberg JI, Walker BD, Campbell TJ (2001) HERG K+ channels: friend or foe. Trends Pharmacol Sci 22:240-246

61. Chiesa N, Rosati B, Arcangeli A, Olivotto M, Wanke E (1997) A novel role for HERG K $K^{+}$channels: spike-frequency adaptation. J Physiol 501:313-318

62. Aronov AM (2005) Predictive in silico modeling for hERG channel blockers. Drug Discov Today 10:149-155

63. Chibale K, Davies-Coleman M, Masimirembwa C (2012) Drug discovery in Africa: impacts of genomics, natural products, traditional medicines, insights into medicinal chemistry, and technology platforms in pursuit of new drugs. Springer, Berlin

64. pan-ANAPL pan-African Natural Products Library [http://www.linkedin.com/ groups/pANPL-4098579/about] Accessed 21 June 2013

doi:10.1186/2191-2858-3-10

Cite this article as: Ntie-Kang et al:: Assessing the pharmacokinetic

profile of the CamMedNP natural products database: an in silico

approach. Organic and Medicinal Chemistry Letters 2013 3:10. 